\title{
Fuzzy Logic Concept for Safety Driven Vehicle- Pedestrian Traffic Interaction
}

\author{
O. M. Olanrewaju \\ Federal University Dutsinma \\ Mathematical Sciences and IT. \\ Dept. \\ P.M.B 5001, Dutsinma \\ Katsina State, Nigeria
}

\author{
A. A. Obiniyi \\ Ahmadu Bello University, Zaria \\ Computer Science \\ Department, , ABU Zaria \\ Kaduna State, Nigeria
}

\author{
S. B. Junaidu \\ Ahmadu Bello University, Zaria \\ Computer Science \\ Department, ABU Zaria \\ Kaduna State, Nigeria
}

\begin{abstract}
In recent times, it has been discovered that fuzzy logic is an important tool for effective traffic control system. Research on intelligent traffic systems has revealed that the implementation of linguistic variables in fuzzy systems help in taking care of diverse possible decisions that can be taken by humans in traffic control. However, the majority of these works focus on vehicular traffic without adequate consideration for pedestrian crossings. This research therefore focuses on incorporation of pedestrian crossing variables into vehicular traffic control using fuzzy logic. The implementation of the fuzzy logic inference system was carried out using MATLAB 2014a. The impact of pedestrian delay, total pedestrian were part of consideration for signal time allocation. The result proved that pedestrian delay has significant contribution to traffic control systems to enhance safety of pedestrians.
\end{abstract}

\section{Keywords}

Fuzzy Logic, Pedestrian, Vehicular traffic control

\section{INTRODUCTION}

Researchers have identified lack of adequate pedestrian facilities, prolonged pedestrian waiting time, human factors (non-compliance drivers and pedestrians) and considerations for vehicular traffic without corresponding attention to pedestrian traffic, among others as major reasons for pedestrian fatalities[5] [1][7]

A social acceptance for an Intelligent Pedestrian Device (IPD) was carried out by [1], who concluded with a recommendation for both the software and hardware implementation of the device. Incorporation of consideration for pedestrian safety which is the subject of this research will be a way forward in meeting this need. The inclusion of the interaction between pedestrians and other parameters in traffic research will enhance safety of pedestrians in traffic streams.

A positive effect of handling pedestrian planning the same way as vehicular traffic is handled, will be an improvement of the safety status of pedestrians walk as a mode of transport.

\section{OVERVIEW OF INTELLIGENT TRANSPORTATION SYSTEMS}

The transfer of human thought and reasoning into technology is the bedrock of intelligent systems. The introduction of this into transport technology gave birth to intelligent transportation systems which is an umbrella term for application information technology to transportation infrastructures and the associated flow entities (Pedestrians and Vehicles).
From literature, it is an established fact that ITS has been used to discover ways of improving safety, reduce congestion and emissions, as well as enhancing productivity. Elements of transportation systems are been embedded with microchips and sensors that enable information collection and communication. This is one of the paths that enhance linkage of transportation system to cloud computing and inter-net of things [4].

Basic attributes of ITS is an advanced adaptive signal control system that performs real-time optimization of traffic signals across a signal network, adjusting signal timing based on prevailing traffic conditions, demand, and system capacity. Conspicuous in the implementation of ITS is case-based reasoning system and fuzzy logic rule based reasoning system.

\subsection{Fuzzy Logic and Intelligent Traffic Control System}

A fuzzy set can be defined as a class with unsharp boundaries. Fuzzy set is a set without rigid sharp boundaries. This means changes from one state to the other is gradual. A fuzzy set A can be defined as:

$A=\{x, \mu A(x) \mid x \in X\}$

Where (x) A $\mu$ is called the membership function (MF) for the fuzzy set A. The MF maps each element of $X$ to a membership value between 0 and 1. (Matlab 2014 manual) By introducing fuzzy logic, the control algorithm will be addressed using rules expressed in human understandable language

This additionally improves robustness of the control algorithms. Fuzzy Logic Control (FLC) has proven effective for complex optimization problems with multiple objectives, uncertain information, and vague decision criteria. Traffic signal timing lies in this category.[2]

\section{FUZZY LOGIC CONTROLLER}

Fuzzification is the process of changing a real scalar value into a fuzzy value. This is achieved with the different types of fuzzifier's membership function. Figure 1 is functional flow structure of the fuzzy logic system. The system receives pedestrian and vehicular traffic inputs from traffic networks for fuzzification, after which the fuzzified values are passed to an inference engine for evaluation using the rule based inference engine, and the resultant fuzzified result is then passed to a deffuzifier for conversion to crisp output which can be used to instruct the traffic actuator. 


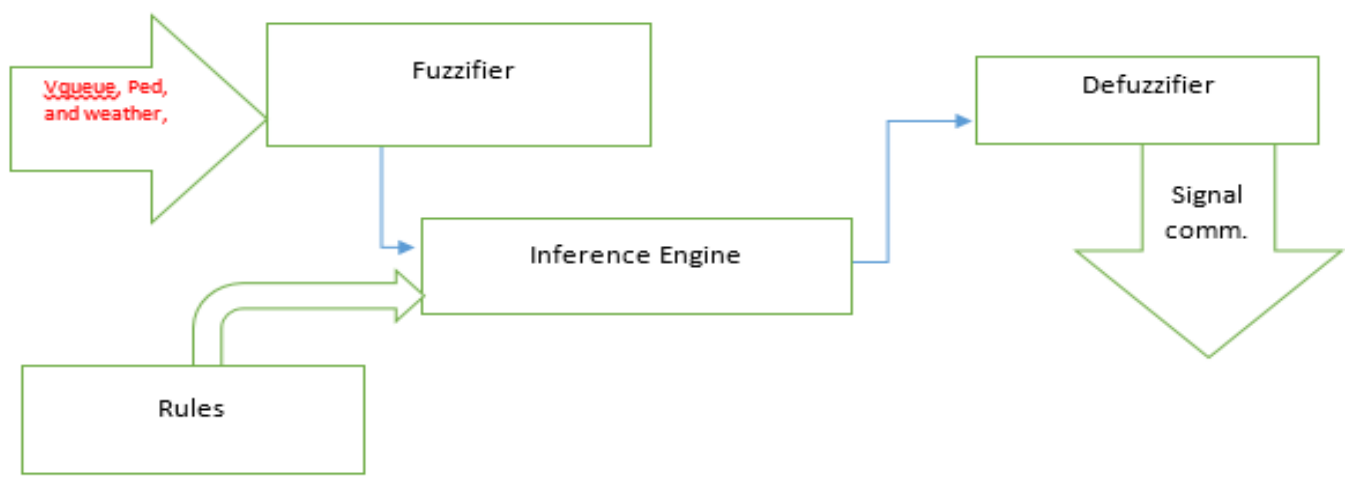

Figure 1: The Fuzzy Logic system for the proposed intelligent traffic control

\subsection{Membership Function}

The interaction between pedestrians and vehicles are constantly been evaluated by total pedestrian volume, vehicle queue and influence of available environmental parameters. Fuzzy logic system fuzzifies the variables' values into linguistic terms according to the membership functions represented in Table 1. The plot of membership functions that shows the overlapping linguistic variables are placed in Figure $2-6$

\subsection{Fuzzy Inference System}

The first step of fuzzy inference -aggregation- determines the degree to which the complete IF-part of the rule is fulfilled. The computation of fuzzy rules is called fuzzy rule inference.
The inference is a calculus consisting of two main steps: aggregation and conclusion.

The inference engine examines the rules (if <conditions > then <actions>) and executes the actions if the information supplied by the user satisfies the conditions in the rules.

The inference method used for this work is for-ward chaining. This is a method involving a top-down approach which accept available facts and draw conclusions based on satisfied rules, this eventually leads to definite intelligent action to be executed. The structure of the inference engine is displayed in Figure 7.

Table 1 Fuzzy Logic Variable and Membership Functions

\begin{tabular}{|c|c|c|c|c|c|c|c|}
\hline \multicolumn{2}{|c|}{ Totalped } & \multicolumn{2}{|l|}{ Ped delay } & \multicolumn{2}{|c|}{ Vqueue } & \multicolumn{2}{|l|}{ Weather } \\
\hline Range & $\begin{array}{l}\text { Linguistic } \\
\text { Value }\end{array}$ & Range & $\begin{array}{l}\text { Linguistic } \\
\text { Value }\end{array}$ & Range & $\begin{array}{l}\text { Linguistic } \\
\text { Value }\end{array}$ & Range & $\begin{array}{l}\text { Linguistic } \\
\text { Value }\end{array}$ \\
\hline $1-9$ & Small & $1-60$ & Low & $1-9$ & Small & Raining & True \\
\hline $7-15$ & Medium & $30-120$ & Medium & $7-30$ & 'medium & Not raining & False \\
\hline $12-60$ & large & $100-600$ & High & $25-50$ & large & & \\
\hline
\end{tabular}

Table 2 Fuzzified Output Variable

\begin{tabular}{|l|}
\hline Signal $\mathrm{cmd} / \mathrm{mem}$. func \\
\hline Short $0-50$ \\
\hline Medium 10-50 \\
\hline Large $40-60$ \\
\hline
\end{tabular}

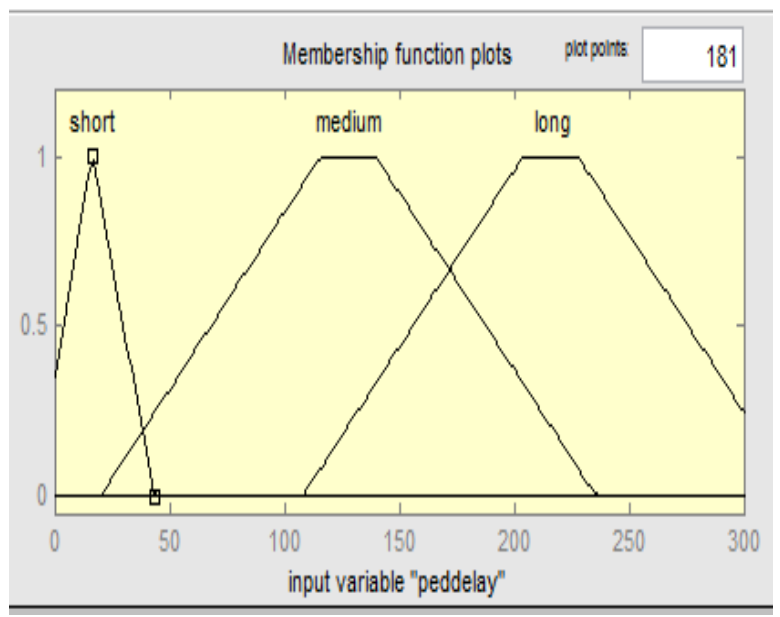

Figure 2: Pedestrian delay plot

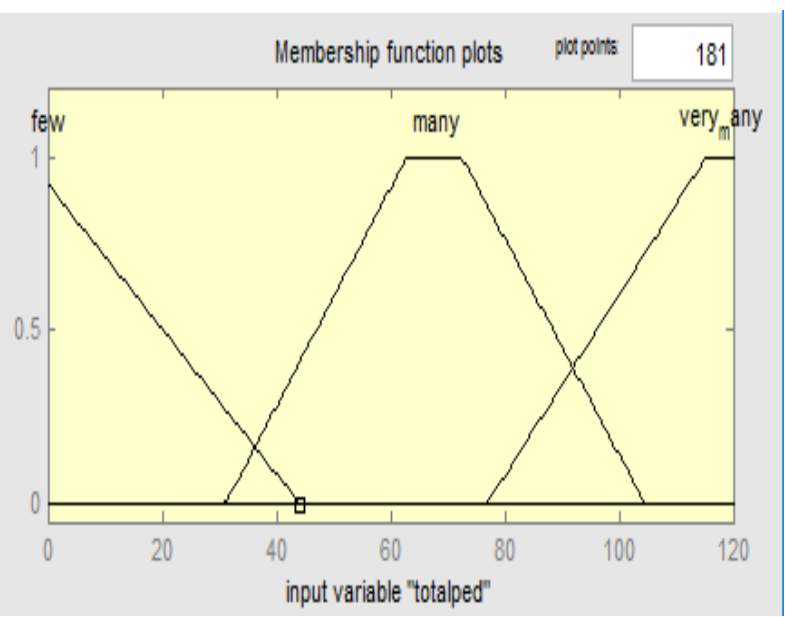

Figure 3: Total pedestrian membership function 


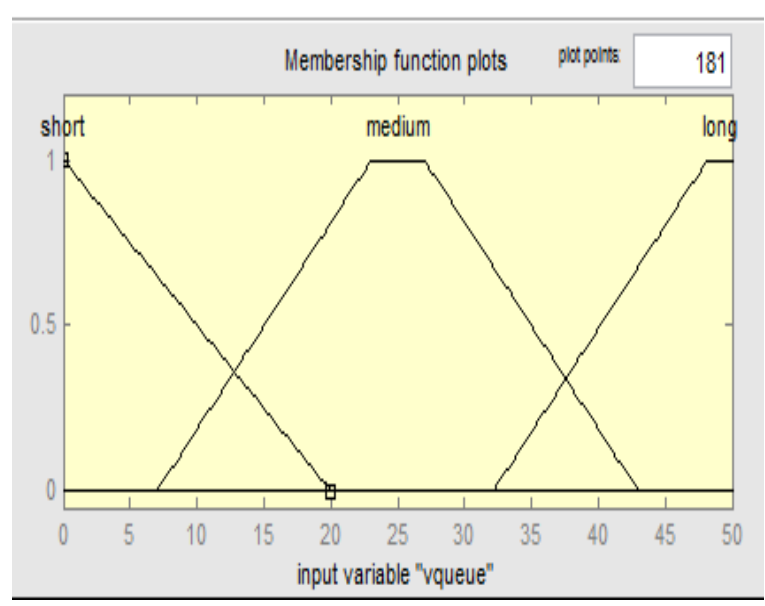

Figure 4: Vehicle Queue

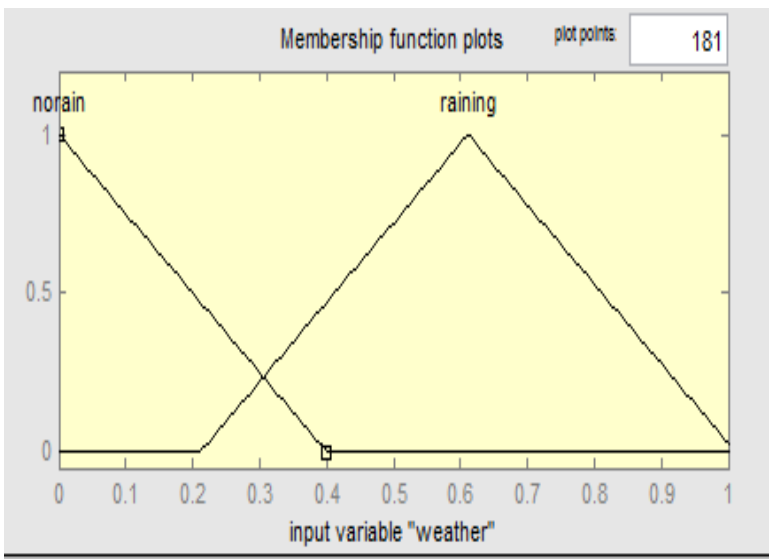

Figure 5: weather membership Function

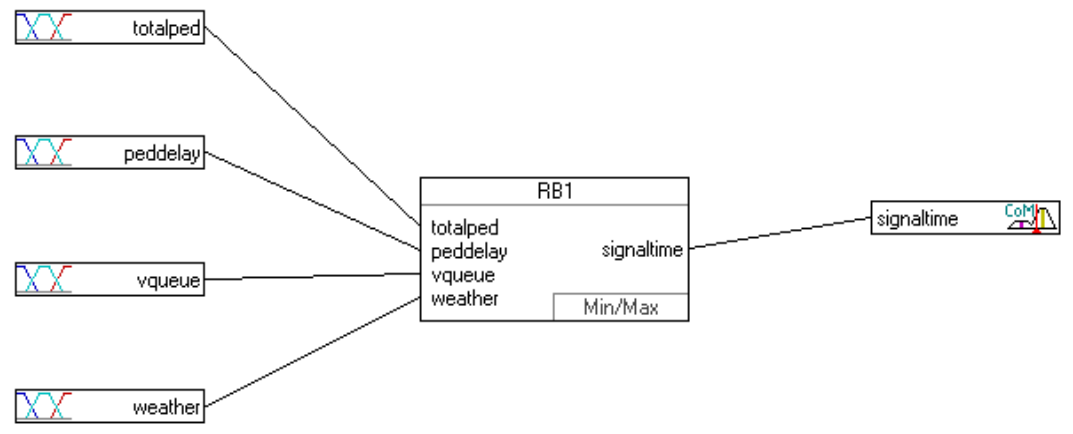

Figure 7 Fuzzy Logic inference System

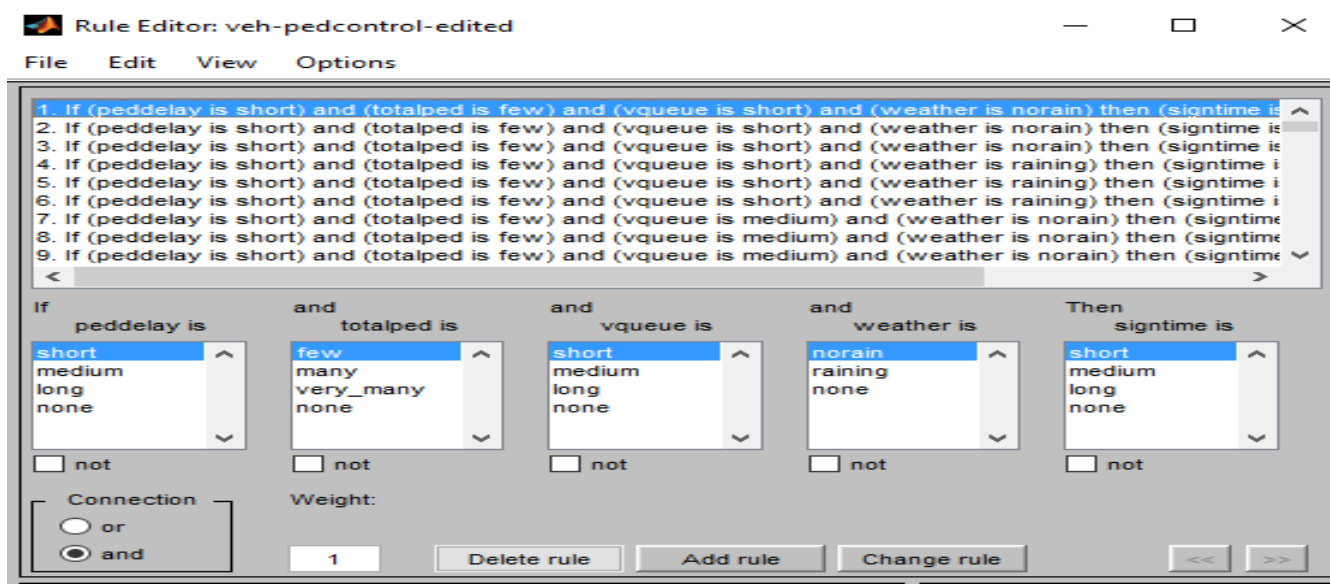

Figure 8: Fuzzy Rule Editor platform 


\section{RESULTS}

Figure 9 is a snapshot of the rule viewer with sample input and signal time output. Table 3 represents various input values with corresponding output values. Figure 10 represents the plot of two input variable at a time with corresponding signal output values. Figure 11 is a line chart used to display trends of signal over pedestrian delay over time. This chart clearly indicates significant consideration for pedestrian delay in signal time control.

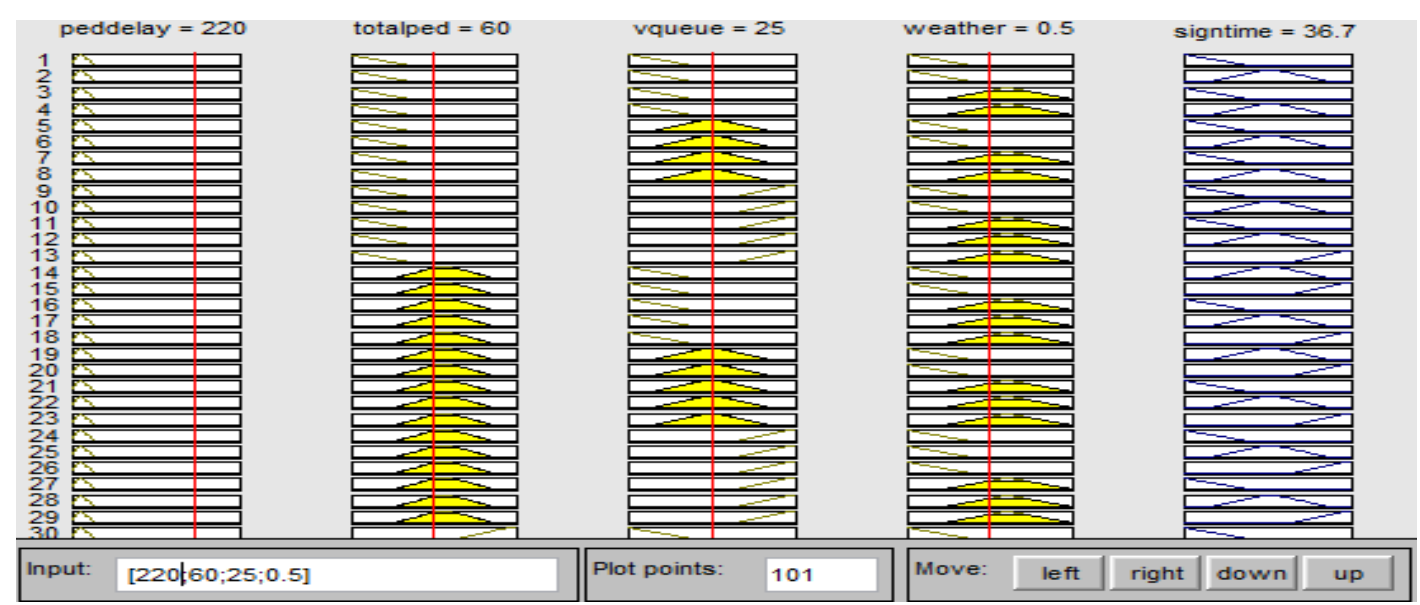

Figure 9: Rule Viewer of the Inference system

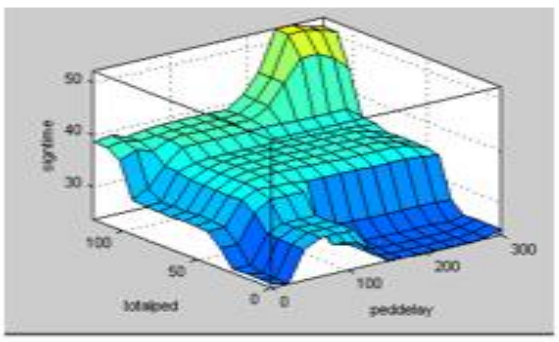

a) Plot of total Ped, peddelay, signaltime

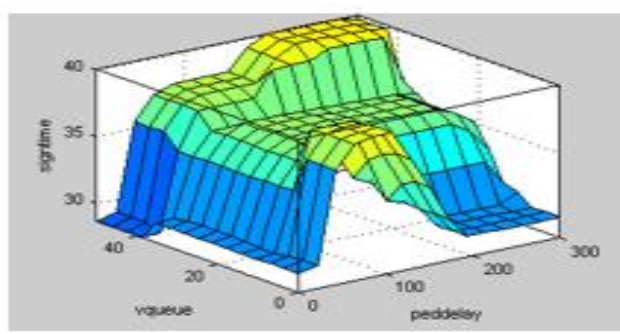

b) Vehqueue, peddelay, signaltime

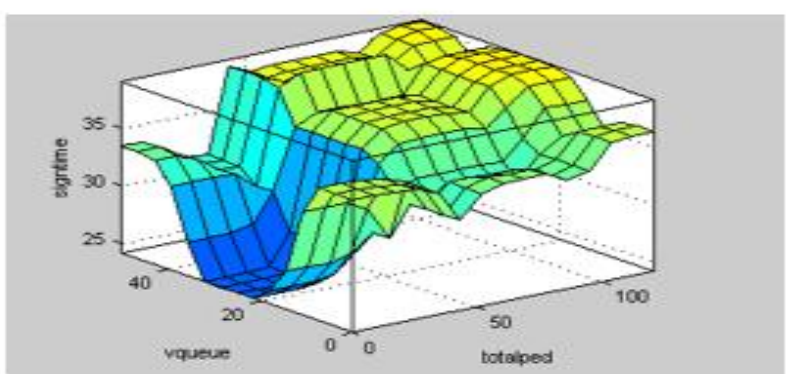

c) vehqueue, total ped and signaltime

Figure 10(a-c): Surface View of Output against selected input variables

Table 3 Sample Output Generated varying Pedestrian delay

\begin{tabular}{|l|l|l|l|l|}
\hline Peddelay(sec) & TotalPed & Vqueue & Weather & $\begin{array}{l}\text { Signaltime } \\
\text { (output) }\end{array}$ \\
\hline 0 & 30 & 25 & 0.5 & 30 \\
\hline 50 & 35 & 25 & 0.5 & 30 \\
\hline 100 & 40 & 25 & 0.6 & 33.3 \\
\hline 150 & 30 & 25 & 0.5 & 34 \\
\hline 200 & 30 & 25 & 0.4 & 34.2 \\
\hline 250 & 30 & 25 & 0.7 & 34.2 \\
\hline
\end{tabular}

\begin{tabular}{|l|l|l|l|l|}
\hline 300 & 37 & 25 & 0.3 & 34.2 \\
\hline 350 & 30 & 25 & 0.5 & 34.2 \\
\hline 400 & 30 & 25 & 0.6 & 35 \\
\hline 450 & 35 & 25 & 0.5 & 35.7 \\
\hline 500 & 35 & 25 & 0.4 & 36 \\
\hline
\end{tabular}




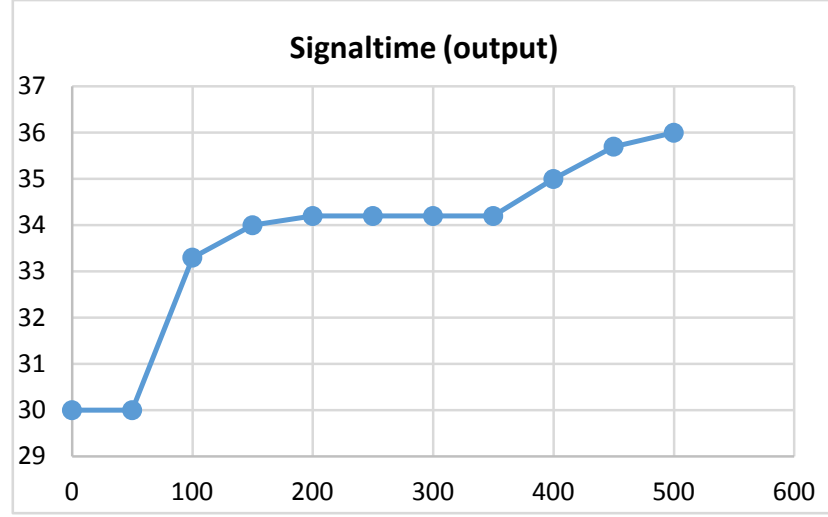

Figure 11: Line chart of signal time against pedestrian delay

\section{CONCLUSION}

This research incorporates pedestrian consideration into signal traffic control system using fuzzy rule based system. The results indicate the necessary impact of incorporating pedestrian variables into traffic signal timing. Pedestrian delay is concluded as an unavoidable variable that must be considered during signalized road traffic control system.

\section{REFERENCES}

[1] Akomolafe B. (2014) Transport Leave a comment daily newswatch,

http://www.mydailynewswatchng.com/author/webofficer-2/ July 17, 2014
[2] Alam J. and Pandey M. K.(2014) Development of Intelligent Traffic Light System Based on Congestion Estimation Using Fuzzy Logic; IOSR Journal of Computer Engineering(IOSR-JCE) Vol. 16 Issue 3, Ver. VI, pp 36-44.

[3] Armsby, Pauline M. (1996) An intelligent pedestriandevice: social, psychological and other issues of feasi-bility. $\mathrm{PhD}$ thesis, Middlesex University. http://eprints.mdx.ac.uk/9847/ retrieved on 10/10/2014.

[4] Aworemi, J. R., Abdul-Azeez, I. A., Olabode, S. O.(2010) Analytical study of the causal factors of road traffic crashes in southwestern Nigeria International Research Journals Vol. 1(4): 118-124 May 2010 http://www.interesjournals.org/ER.

[5] Papacostas C.S. and Prevedouros P. D.(2008) Transporation Engineering and Planning, third Edition, PrenticeHall of India Private Limited.

[6] Parulekar G.D., Desai D. B., and Gupta A. K. An Overview of "Intelligent Transportation System": Transportation Management through Modern Technology- A Survey IOSR Journal of Mechanical and Civil Engineering (IOSR-JMCE) ISSN: 2278-1684, PP: 33-34 www.iosrjournals.org

[7] Van-Houten, R., Malenfant, L., Blomberg,R. D., Huitema B. E., and Casella, S. (2013). High-Visibility Enforcement on Driver Compliance with Pedestrian Right-of-Way Laws. (Report No. DOT HS 811 786). Washington, DC: National Highway Traffic Safety Administration. 